\title{
Clinical Course and Outcome of Critically Ill Obese Patients with COVID-19 Admitted in Intensive Care Unit of a Single Center: Our Experience and Review
}

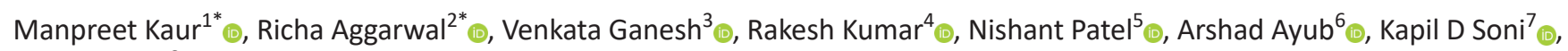 \\ Anjan Trikha ${ }^{8}$
}

\begin{abstract}
Introduction: Obesity has been considered as one of the independent risk factors for a severe form of coronavirus disease-2019 (COVID-19) and relationship between obesity, critical illness, and infection is still poorly understood. We herein discuss clinical course and outcome of critically ill obese patients with COVID-19 admitted to critical care unit.

Materials and methods: We retrospectively analyzed data of critically ill obese patients hospitalized with COVID-19 over a span of 6 months. Management was guided according to the institutional protocol. Collected data included demographic parameters (age, sex, comorbidities, and body mass index (BMI)), complications, inflammatory markers (interleukin (IL)-6, Ferritin), length of mechanical ventilation, length of intensive care unit (ICU) stay, and inhospital death.

Results: There was no appreciable difference in terms of demographics, inflammatory markers, predictors of mortality scores, and comorbidity indices between the survivors and nonsurvivors. Among outcome analysis, there was a statistically significant difference between ventilator days between survivors and nonsurvivors $\left(p=0.003^{* *}\right)$.

Conclusion: Obesity itself is a significant risk factor for severe COVID-19 infection; however, if efficiently managed and in a protocol-determined manner, it can have a favorable outcome.

Keywords: Coronavirus disease-2019, Obesity, Pandemic, Predictors of mortality.

Indian Journal of Critical Care Medicine (2021): 10.5005/jp-journals-10071-24047
\end{abstract}

\section{BACKGROUND}

Globally, prevalence of obesity has risen and hence more patients with obesity are likely to be admitted with coronavirus disease-2019 (COVID-19). There is a complicated relationship between obesity, critical illness, and infection which is poorly understood. Obese people are at higher risk of developing severe symptoms, hospitalization, venous thromboembolism, dialysis, mechanical ventilation, and inhospital death. ${ }^{2}$

There is literature from COVID-19 obese patients from the Western countries but there is a paucity of literature available from the Indian subcontinent. We herein describe the clinical course and outcome analysis of obese patients with COVID-19 admitted to intensive care unit (ICU) of a single center and discuss the challenges faced.

\section{Materials and Methods}

\section{Study Design and Patients}

We retrospectively analyzed data of critically ill obese patients hospitalized with COVID-19 over a span of 6 months from August 2020 to January 2021 after local ethics committee approval. Inclusion criteria were as follows: patients admitted to ICU with a confirmed diagnosis of acute COVID-19 infection and patients categorized as obese with body mass index (BMI) $\geq 30$ according to the World Health Organization (WHO) classification.

\section{Data Collection and Evaluation}

Data collection was performed by clinical research anesthesia faculty who reviewed their medical files and extracted the data.

\begin{abstract}
1,4,5 Department of Anaesthesiology, Pain Medicine and Critical Care, All India Institute of Medical Sciences, New Delhi, India

${ }^{2}$ Department of Critical Care, All India Institute of Medical Sciences, New Delhi, India

3,6,8 Department of Anaesthesia, All India Institute of Medical Sciences, New Delhi, India

${ }^{7}$ Department of Critical and Intensive Care, JPN Apex Trauma Centre, All India Institute of Medical Sciences, New Delhi, India

* Manpreet Kaur and Richa Aggarwal are co-first author for this article

Corresponding Author: Nishant Patel, Department of Anaesthesiology, Pain Medicine and Critical Care, All India Institute of Medical Sciences, New Delhi, India, Phone: +91 8510955882, e-mail: pateldrnishant@ gmail.com
\end{abstract}

How to cite this article: Kaur M, Aggarwal R, Ganesh V, Kumar R, Patel N, Ayub A, et al. Clinical Course and Outcome of Critically III Obese Patients with COVID-19 Admitted in Intensive Care Unit of a Single Center: Our Experience and Review. Indian J Crit Care Med 2021;25(12):1382-1386.

Source of support: Nil

Conflict of interest: None

Subjects were categorized according to the Centers for Disease Control (CDC) classification as follows: class I (BMl of $30-34.9 \mathrm{~kg} / \mathrm{m}^{2}$ ), class II (BMI of $35-39.9 \mathrm{~kg} / \mathrm{m}^{2}$ ), and class III (BMI of $40 \mathrm{~kg} / \mathrm{m}^{2}$ or higher). Collected data included demographic characteristics (age, sex, height, weight, and BMI), microvascular and macrovascular 
complications and comorbidities, inflammatory markers (IL-6, Ferritin), length of mechanical ventilation, length of ICU stay, inhospital death, adverse cardiovascular, and renal and venous thromboembolic outcomes.

\section{Institutional Protocol}

As per the institutional protocol, we administered antibiotics according to the antibiotic stewardship, methylprednisolone $0.5-1 \mathrm{mg} / \mathrm{kg}$ (moderate disease), $1-2 \mathrm{mg} / \mathrm{kg}$ (severe disease). Patients with moderate-to-severe disease were administered remdesivir $200 \mathrm{mg}$ IV followed by $100 \mathrm{mg}$ IV for 5 days early especially if there was an increasing oxygen requirement. We used Enoxaparin $1 \mathrm{mg} / \mathrm{kg}$ body weight/dalteparin 2500 units BD subcutaneously for anticoagulation. Oxygen delivery at our institution was to target $\mathrm{SpO}_{2} \geq 92 \%$, stepping up from high-flow nasal cannula (HFNC) to noninvasive and if need be, to mechanical ventilation. Early proning was done (both nonventilated and ventilated patients) whenever feasible.

\section{Statistical Analysis}

Data were analyzed using IBM SPSS Statistics for Windows, version 25.0 (IBM Corp., Armonk, New York, USA, 2017). Descriptive data were presented as counts, mean $\pm S D$, and median numbers where appropriate. For all relevant statistics, we assumed a $p<0.05$ as significant. About $4.012 \%$ of data were missing as some inflammatory markers were not available owing to laboratory constraints. Little's MCAR test was not significant ( $p$ value of 0.446 ), suggesting that data were missing completely at random. Since these missing data were less than $5 \%$ and multiple imputation did not add any significant change in terms of pooled values, we proceeded with analyzing the raw data. Normally distributed numerical variables were analyzed using independent samples $t$-test/Mann-Whitney $U$ test where appropriate. Categorical variables were analyzed using Chi-squared/Fisher's exact test where appropriate. Relevant odds ratios were calculated.

\section{Results}

The number of obese patients according to CDC classification were as follows: nine (50\%) in class I, six (22.22\%) in class II, and three (11.11\%) in class III. Associated comorbidities were hypertension in six (33.33\%), diabetes in six (33.33\%), hypothyroidism in three (16.6\%), asthma in one (5.55\%), chronic kidney disease (CKD) in one $(5.55 \%)$, coronary artery disease (CAD) in one $(5.55 \%)$, and obstructive sleep apnea (OSA) in one (5.55\%). With respect to gender, 11 were males and 7 were female. Presenting chief complaints were shortness of breath in 17 (94\%), fever in 11 (61.11\%), cough in 10 (55.5\%), and altered sensorium in 2 (11.11\%). Detailed demographics and clinical and laboratory parameters are summarized in Table 1. The mean initial sequential organ failure assessment (SOFA) score was 3 (2-4.5). The initial respiratory support required in patients varied. Among them, 1 (5.5\%) needed only non-rebreathing mask, 4 (22.2\%) required HFNC alone, and 13 $(72.2 \%)$ required alternate HFNC and noninvasive ventilation (NIV). Ten out of 18 (55\% obese) needed invasive ventilation later in their course. Among treatment modalities, 14 patients (77.77\%) were administered remdesivir, 3 (16.66\%) convalescent plasma, and all received methylprednisolone. The median length of ICU stay was 11.5 , median ventilator days were 1.5 , and length of hospital stay was 20 . Out of 18 patients, 8 did not survive. There was no appreciable difference in terms of demographics, inflammatory
Table 1: Demographics and clinical and laboratory features of the obesity cohort

\begin{tabular}{|c|c|}
\hline & $N=18$ \\
\hline Age in years & $53(41.5-60)$ \\
\hline Weight in kg & $94.5(87.25-98.75)$ \\
\hline Height in meters & $1.65(1.59-1.69)$ \\
\hline $\mathrm{BMI} \mathrm{kg} / \mathrm{m}^{2}$ & $35.33(33.17-39.34)$ \\
\hline \multicolumn{2}{|c|}{ Baseline laboratory parameters } \\
\hline IL-6 (pg/mL) & $33.94(14.93-129.42)$ \\
\hline Ferritin $(\mathrm{ng} / \mathrm{mL})$ & $435.8(319-1166.6)$ \\
\hline CRP (mg/L) & $10.4(4.98-24.78)$ \\
\hline D-dimer $(\mu \mathrm{g} / \mathrm{mL})$ & $0.58(0.42-0.65)$ \\
\hline Platelets $(/ \mu \mathrm{L})$ & $216,000(172,500-263,750)$ \\
\hline Bilirubin (mg/dL) & $0.7(0.6-1.125)$ \\
\hline Creatinine (mg/dL) & $0.915(0.78-1.43)$ \\
\hline \multicolumn{2}{|c|}{$\begin{array}{l}\text { Laboratory parameters at } 48 \text { hours } \\
\text { of admission }\end{array}$} \\
\hline Platelet $(/ \mu \mathrm{L})$ & $243,000(182,500-317,250)$ \\
\hline Bilirubin (mg/dL) & $0.9(0.68-1.43)$ \\
\hline Creatinine (mg/dL) & $0.95(0.6-1.63)$ \\
\hline \multicolumn{2}{|l|}{ Temporal parameters } \\
\hline ICU stay (in days) & $11.5(8.25-19)$ \\
\hline Ventilator days & $1.5(0-8)$ \\
\hline Hospital stay & $20(10.75-24.25)$ \\
\hline \multicolumn{2}{|l|}{ Severity indices } \\
\hline SOFA at admission & $3(2-4.5)$ \\
\hline SOFA at 48 hours & $3.5(2-5.25)$ \\
\hline Charlson Comorbidity Index & $2(1-3)$ \\
\hline
\end{tabular}

Median (IQR) is presented

markers, predictors of mortality scores, and comorbidity indices between the survivors and nonsurvivors which are summarized in Table 2.

Males appeared to have a higher odds of death compared to females (OR $5,0.442-56.623, p=0.194$ ) but was statistically insignificant owing to the small sample size. Mortality was also high among those who received invasive ventilation (60\% mortality, OR 24.57, 1.11-542.44, $p=0.042$ ) compared to those who did not (100\% survived). Figure 1 shows a simple boxplot of ventilator days against outcome of discharge and death. The generalizability of this finding is, however, questionable due to a wide $95 \%$ confidence interval.

\section{Discussion}

Obesity is linked to low-grade inflammation which in turn contributes to multiple metabolic diseases (dyslipidemia, insulin resistance, and diabetes mellitus (DM)) and modifies innate and adaptive immunity, thereby increasing susceptibility to infections. It decreases responsiveness to antivirals and antimicrobial drugs. ${ }^{3}$ Obesity is an important driver of hospitalization as obese patients infected with COVID-19 develop severe disease. We herein share clinical course and outcome of critically ill obese patients with COVID-19 of a single center from the Indian subcontinent.

Age and weight in our cohort were like the demographic parameters of the largest obese COVID-19 cohort by Henderen et al. from Western literature. The age in COVID-19 obese in their cohort ranged from 41 to 73 years compared to 53 (41.5-60 years) 
Table 2: Similarities and differences between survivors and nonsurvivors

\begin{tabular}{|c|c|c|c|}
\hline & Nonsurvivors $(n=6)$ & Survivors $(n=12)$ & pvalue \\
\hline Age in years & $49 \pm 9.19$ & $52.33 \pm 14.91$ & 0.618 \\
\hline Weight in kg & $106.83 \pm 31.87$ & $95 \pm 19.87$ & 0.329 \\
\hline Height in meters & $1.67 \pm 0.09$ & $1.61 \pm 0.14$ & 0.553 \\
\hline $\mathrm{BMI} \mathrm{kg} / \mathrm{m}^{2}$ & $37.57 \pm 6.30$ & $36.71 \pm 4.88$ & 0.75 \\
\hline \multicolumn{4}{|l|}{ Baseline laboratory parameters } \\
\hline IL-6 (pg/mL) & $56.72 \pm 54.40$ & $77.70 \pm 74.35$ & 0.607 \\
\hline Ferritin $(\mathrm{ng} / \mathrm{mL})$ & $609.46 \pm 437.42$ & $674.71 \pm 498.65$ & 0.456 \\
\hline $\mathrm{CRP}(\mathrm{mg} / \mathrm{L})$ & $15.84 \pm 7.67$ & $11.10 \pm 8.18$ & 0.24 \\
\hline D-dimer $(\mu \mathrm{g} / \mathrm{mL})$ & $0.54 \pm 0.11$ & $0.54 \pm 0.10$ & 1 \\
\hline Platelets $(/ \mu \mathrm{L})$ & $194000 \pm 97621.72$ & $223916.66 \pm 64220.44$ & 0.75 \\
\hline Bilirubin (mg/dL) & $0.81 \pm 0.28$ & $0.90 \pm 0.51$ & 0.964 \\
\hline Creatinine (mg/dL) & $1.46 \pm 1.15$ & $1.06 \pm 0.48$ & 0.437 \\
\hline \multicolumn{4}{|l|}{$\begin{array}{l}\text { Laboratory parameters at } 48 \text { hours } \\
\text { of admission }\end{array}$} \\
\hline Platelet $(/ \mu \mathrm{L})$ & $216166.67 \pm 8386.55$ & $275000 \pm 128631.26$ & 0.494 \\
\hline Bilirubin (mg/dL) & $0.95 \pm 0.43$ & $1.08 \pm 0.53$ & 0.616 \\
\hline Creatinine (mg/dL) & $1.18 \pm 0.84$ & $1.35 \pm 1.13$ & 0.682 \\
\hline \multicolumn{4}{|l|}{ Temporal parameters } \\
\hline ICU stay (in days) & $18.83 \pm 10.70$ & $11.17 \pm 5.36$ & 0.125 \\
\hline Ventilator days & $10.67 \pm 8.02$ & $1.58 \pm 2.75$ & $0.003^{* *}$ \\
\hline Hospital stay & $20 \pm 11.59$ & $17.92 \pm 7.09$ & 0.862 \\
\hline \multicolumn{4}{|l|}{ Severity indices } \\
\hline SOFA at admission (Median, IQR) & $3.5(3-5.25)$ & $3(2-5.5)$ & 0.437 \\
\hline SOFA at 48 hours (Median, IQR) & $4.5(3-6.25)$ & $3(2-4.75)$ & 0.18 \\
\hline $\begin{array}{l}\text { Charlson Comorbidity Index } \\
\text { (Median, IQR) }\end{array}$ & $1(0-2)$ & $2(1.25-3)$ & 0.053 \\
\hline
\end{tabular}

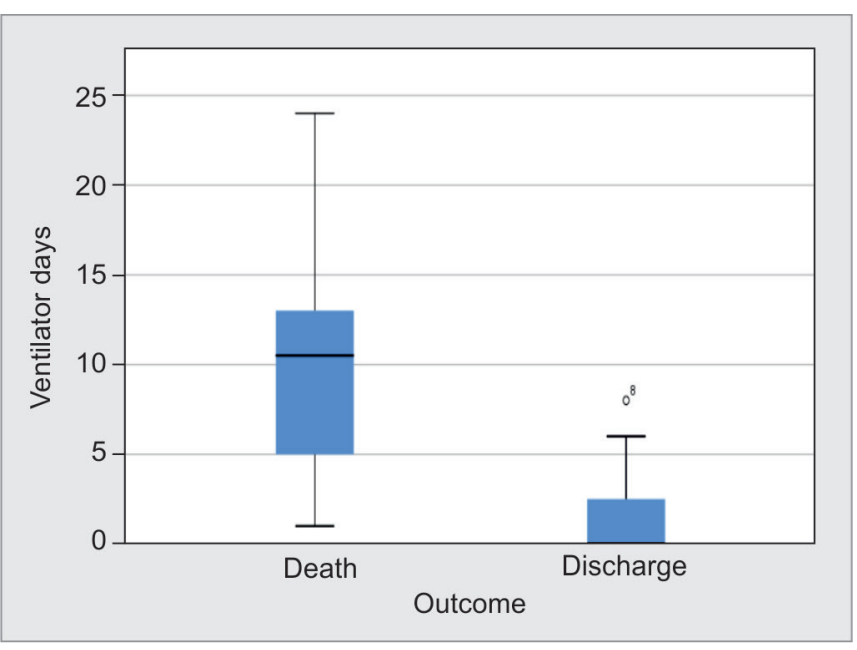

Fig. 1: Ventilator days against the outcome of discharge and death

years in our study. The weight range was wide from 83 to $146 \mathrm{~kg}$ compared to $94.5(87.25-98.75)$ in ours. Their study cohort had male predominance $(55 \%)$ like our cohort which was $61 \%$. Males tend to have more visceral fat which can induce an inflammatory response and raised C-reactive protein (CRP) compared to females who have more subcutaneous fat. ${ }^{4}$ Henderen et al. found a high prevalence of hypertension (59.2-63\%) and diabetes (40.4-42\%) as the most significant comorbidity associated with obesity. ${ }^{2}$ In our study as well, diabetes and hypertension were the commonest comorbidities but had slightly lower incidence of 33\% each. The incidence of other comorbidities like CKD, asthma, and CAD was lesser in our cohort with each being $5.5 \%$ compared to the Western cohort where it was 9.3-11, 10-17.7, and 17-19\%, respectively. The proportions of each BMI category in the American Heart Association (AHA) COVID-19 cardiovascular disease study by Henderen et al. were as follows: class I obesity (21\%), class II obesity (11\%), and class III obesity (11\%), while at our center were $50,22.22$, and $11.11 \%$ in class I, class II, and class III obesity, respectively. ${ }^{2}$

COVID causes severe respiratory damage in obese. Hence, a majority of obese patients presented with shortness of breath and oxygen desaturation on admission and only a minority had neurological involvement. The above-mentioned factors also make the COVID-infected obese more at risk of developing pneumonia. In Hendren et al.'s study, complaints in patients were higher prevalence of shortness of breath (76.2-76.9\%) and fever (65.1-68.5\%) like our cohort. ${ }^{2}$

HFNC is the preferred initial choice at our center as it provides high oxygen concentrations compared to the conventional methods of oxygenation. HFNC creates positive expiratory pressure and reduces dead space. It is known that mortality of invasively ventilated COVID patients is high and has difficult extubation. 
Hence, it is prudent to use HFNC as the initial choice which we alternated with NIV as and when needed. Even the National Institutes of Health (NIH) Panel recommends HFNC over NIV (BIla) in adults with COVID-19 and acute hypoxemic respiratory failure. ${ }^{5}$ NIV may be beneficial in respiratory failure in COPD exacerbations and in acute cardiogenic pulmonary edema. ${ }^{4}$ Among the cohort, 1 (5.5\%) needed only non-rebreathing mask, 4 (22.2\%) required HFNC alone, and 13 (72.2\%) required alternate HFNC and NIV. However, 10 out of 18 (55.5\%) needed NIV later.

While using any mode of oxygenation, anesthesiologist might have to confront several challenges (rapid desaturation, difficult ventilation, and intubation), and hence, difficult airway cart should be readily available at the bedside.

We used remdesivir in 14 patients (77.77\%) early as part of our institutional protocol and its dosage is not weight based. We used convalescent plasma in $11 \%$ of the patients. ${ }^{2}$ Administration of remdesivir upon increasing oxygen requirement highlights the greater respiratory disease severity with obesity.

There was no statistical difference in demographics and severity indices (admission SOFA, SOFA at 48 hours, and Charlson Comorbidity Index) among survivors and nonsurvivors of COVID obese patients (Table 2). Severity of lung infection in COVID-19 is due to cytokine storm and immune hyperactivation. Obesity produces proinflammatory milieu as adipocyte hypoxia causes exaggerated cytokine release tumor necrosis factor a (TNF- $\alpha$ ), IL-1 $\beta$ and IL-6, and immune cells recruitment creating an autoregenerating inflammation loop. ${ }^{6}$ Study by McNeill found that obese individuals had higher initial and peak levels of CRP and ESR, d-dimer but similar IL-6 and ferritin compared to nonobese. Greater disease severity in obese is characterized by more exuberant inflammation. ${ }^{7}$ In our cohort of obese patients, the inflammatory markers, IL-6, ferritin, and CRP were raised, as multiples of lower limits of normal, by 25.9, 97.2, and 49.6 times, respectively.

Among the outcome analysis, there was a statistically significant difference between ventilator days between survivors and nonsurvivors. It is well known that mortality in COVID-19 patients requiring invasive ventilation is high and it holds true for obese COVID patients as well. ${ }^{8}$ However, there was no statistically significant difference between duration of ICU stay and duration of hospital stay in between survivors and nonsurvivors. It could be attributed to the small sample size of the study population.

Among the 18 patients in the cohort, 12 (66\%) have been transferred alive from the ICU, 6 died (33\%) in the ICU all of whom were mechanically ventilated with the average days of ventilator days $10.67 \pm 8.02$. Among patients who received invasive mechanical ventilation, ICU mortality was $60 \%(6 / 10)$. Comorbidities like diabetes increase the likelihood of severe illness. ${ }^{9}$

Multiple problems are faced by the intensivists in dealing with obese COVID patients. One may need specialized beds for obese patients keeping in mind the maximum weight-bearing capacity of the intensive care bed. Positioning them is a daunting task and needs manpower to achieve it. Hence, taking a chest roentgenogram and proning them is very difficult. Besides, proning increases airway pressures and practically it is easier to achieve a lateral position compared to the prone position in morbidly obese. They are more likely to develop pressure sores due to a huge weight on the dependent areas.

Point-of-care ultrasonography (POCUS) is an important tool in COVID-19 settings as auscultation is not possible and patient transfer to the radiology suite is tough. But ultrasound beam must penetrate a large subcutaneous fat, thereby compromising the quality of POCUS.

Obesity causes low-grade inflammation and impaired fibrinolysis increasing the likelihood of thrombosis and generalized thrombotic microvascular injury, potentially worsening lung damage. Hence, heparin should be used for both prophylactic and therapeutic use in different COVID-19 protocols. We used inj Enoxaparin $1 \mathrm{mg} / \mathrm{kg}$ body weight subcutaneously for anticoagulation and inj methylprednisolone $0.5-1 \mathrm{mg} / \mathrm{kg}$ in moderate disease and $1-2 \mathrm{mg} / \mathrm{kg}$ body weight in severe cases. Drug dosage adjustment needs to be taken into consideration for drugs like remdesivir (fixed dose), steroid, Clexane, and tocilizumab. COVID-19 obese patients are at increased risk of severe disease, metabolic dysfunction, and worse outcomes. Aggressive management should be done in obese individuals regardless of age, and they should be prioritization for a COVID-19 vaccine in obese patients.

Limitations of our discourse are that it was a retrospective analysis with a small sample size from a single ICU.

\section{Conclusion}

Awareness about problems and challenges faced in managing COVID obese patients can strengthen the healthcare system. Although obesity is itself considered a significant risk factor for severe COVID-19 infection; however if efficiently managed, it can have a favorable outcome as seen in our obese COVID-19 cohort.

\section{ACKNOWLEDGMENTS}

Authors would like to thank Dr Manpreet Kaur and Dr Richa Aggarwal because both have contributed equally in this study.

\section{ORCID}

Manpreet Kaur (1) https://orcid.org/0000-0003-3069-1562 Richa Aggarwal (1) https://orcid.org/0000-0002-4531-2759 Venkata Ganesh (1) https://orcid.org/0000-0001-8265-5198 Rakesh Kumar (i) https://orcid.org/0000-0002-1539-8264 Nishant Patel (1) https://orcid.org/0000-0001-9376-4963 Arshad Ayub 이 https://orcid.org/0000-0002-8441-2509 Kapil D Soni @ https://orcid.org/0000-0003-1214-4119 Anjan Trikha $\odot$ https://orcid.org/0000-0002-6001-8486

\section{References}

1. More is not better: the complicated relationship between obesity, critical illness, and infection - PubMed. 2021. Available from: https:// pubmed.ncbi.nlm.nih.gov/33638648/.

2. Hendren NS, de Lemos JA, Ayers C, Das SR, Rao A, Carter S, et al. Association of body mass index and age with morbidity and mortality in patients hospitalized with COVID-19: results from the American Heart Association COVID-19 Cardiovascular Disease Registry. Circulation 2021;143(2):135-144. DOI: 10.1161/ CIRCULATIONAHA.120.051936.

3. Dhurandhar NV, Bailey D, Thomas D. Interaction of obesity and infections. Obes Rev 2015;16(12):1017-1029. DOI: 10.1111/ obr.12320.

4. Tsao Y-C, Chen J-Y, Yeh W-C, Li W-C. Gender- and age-specific associations between visceral obesity and renal function impairment. Obes Facts 2019;12(1):67-77. DOI: 10.1159/000496626. 
5. Gürün Kaya A, Öz M, Erol S, Çiftçi F, Çiledağ A, Kaya A. High flow nasal cannula in COVID-19: a literature review. Tuberk Toraks 2020;68(2):168-174. DOI: 10.5578/tt.69807.

6. Muscogiuri G, Pugliese G, Barrea L, Savastano S, Colao A. Commentary: obesity: the "Achilles heel" for COVID-19? Metabolism 2020;108:154251. DOI: 10.1016/j.metabol.2020.154251.

7. McNeill JN, Lau ES, Paniagua SM, Liu EE, Wang JK, Bassett IV, et al. The role of obesity in inflammatory markers in COVID-19 patients. Obes Res Clin Pract 2021;15(1):96-99. DOI: 10.1016/j.orcp.2020.12.004.
8. King CS, Sahjwani D, Brown AW, Feroz S, Cameron P, Osborn E, et al. Outcomes of mechanically ventilated patients with COVID-19 associated respiratory failure. PLoS One 2020;15(11):e0242651. DOI: 10.1371/journal.pone.0242651.

9. Smati S, Tramunt B, Wargny M, Caussy C, Gaborit B, Vatier C, et al. Relationship between obesity and severe COVID-19 outcomes in patients with type 2 diabetes: results from the CORONADO study. Diabetes Obes Metab 2021;23(2):391-403. DOI: 10.1111/dom. 14228. 